\title{
ESTADO DEL ARTE DE LOS MÉTODOS PARA DETECCIÓN DE PESTICIDAS ORGANOCLORADOS EN FRUTAS
}

\section{STATE OF THE ART METHODS FOR DETECTION OF ORGANOCHLORINE PESTICIDES IN FRUITS}

Maldonado O. Yohanna ${ }^{1 *}$, Maldonado-M. Lida ${ }^{1}$, Rivera María E. ${ }^{2}$, Caballero P. Luz A. ${ }^{1}$

${ }^{1}$ Universidad de Pamplona, Maestría en Ciencia y Tecnología de Alimentos. Grupo de Investigación GIBA. Km.1 Vía Bucaramanga, Pamplona, Colombia. *johamalob@gmail.com.

${ }^{2}$ Facultad de Ingenierías y Arquitectura. Departamento Civil y Ambiental. Grupo de Investigación Ambientales Agua, Aire y Suelo (GIAAS). Universidad de Pamplona.

Pamplona. Colombia.

Recibido 21 de Julio 2016; aceptado 30 de Noviembre de 2016

\section{RESUMEN}

El estudio de los residuos de plaguicidas presentes en alimentos ha sido una tarea de investigación constante y cada vez el monitoreo analítico cobra mayor importancia, está más normalizado y demanda técnicas de seguimiento in situ y en tiempo real. Entre los cuales los biosensores, constituyen una herramienta de gran precisión y exactitud para los sistemas de monitoreo. Por lo anterior, esta revisión pretende mostrar algunos desarrollos en el tratamiento de la muestra y en complementos instrumentales para la optimización de los procesos de identificación y cuantificación de contaminantes en matrices alimenticias. Para tal fin, se relacionan las técnicas utilizadas, métodos de extracción y tendencias 
Autor a quien dirigirse la correspondencia: Maldonado O. Yohanna Correo electrónico: johamalob@gmail.com. alternativas innovadoras para el análisis de estos compuestos. Finalmente se incluyen las investigaciones realizadas en este tema en el área de alimentos y los avances que se han desarrollado para mejorar las técnicas de análisis.

Palabras clave: Frutas, métodos de extracción. pesticidas, sistema multisensorial.

\section{ABSTRACT}

The study of residues of pesticides present in food has been a constant research task and increasingly analytical monitoring is more important, more standardized and requires on-site and real-time monitoring techniques. Among them the biosensors, constitute a tool of great precision and accuracy for the systems of monitoring. Therefore, this review aims to show some developments in the sample treatment and in instrumental complements for the optimization of the identification and quantification processes of contaminants in food matrices. For this purpose, the techniques used, extraction methods and innovative alternative trends for the analysis of these compounds are related. Finally, the research carried out in this area in the area of food and the advances that have been developed to improve the techniques of analysis are included.

Key words: fruits, extraction methods, pesticides, multisensory system.

\section{INTRODUCCIÓN}

Durante décadas el hombre ha desarrollado la agricultura como una de sus principales fuentes alimentarias, motivo por el cual busca mejorar las condiciones de productividad y calidad de las cosechas, para lo cual se han tenido que implementar modelos de producción agrícola de alto rendimiento para suplir con las necesidades y exigencias que día a día van surgiendo en el mercado. Existen diferentes estrategias para aumentar la 
productividad alimentaria como: nuevas técnicas agrícolas, el empleo de semillas mejoradas y el uso de productos de protección de cultivos, grupo dentro del cual están los plaguicidas (Guerrero, 2003; Moreno, 2001). Cuando se aplica una o varias de estas estrategias combinadas, puede llegar a multiplicarse la cosecha normal de un cultivo.

Colombia es un país agrícola por excelencia y el uso de plaguicidas se ha convertido en una necesidad básica en los cultivos de frutas y hortalizas como una herramienta eficaz para controlar enfermedades, insectos, malezas y otros organismos que pueden interferir con la producción de cultivos. La utilización de diversos productos químicos en la producción agrícola para controlar las plagas y enfermedades, así como para disminuir los riesgos y pérdidas de los sistemas agrícolas, ha sido un reto permanente (Sánchez, 2002), ya que su uso se debe a las propiedades biocidas y de selectividad que poseen constituyéndose como el método habitual de lucha contra las plagas. (Cooper \& Dobson, 2007; Cortes, Sánchez, DíazPlaza, Villen, \& Vásquez, 2006).

El uso inadecuado de los plaguicidas particularmente la sobredosificación, además de su aplicación en tiempos no apropiados y en cultivos en los que no han sido registrados, hacen de estas sustancias, un riesgo potencial para la salud humana y para el medio en general, ya que se trata de productos generalmente tóxicos, que pueden presentar residuos contaminantes en el producto final que va al consumidor y por ende disminución en su calidad (Muñoz, 1992). Debido a que una de las prácticas agrícolas más frecuentes es el monocultivo no tecnificado y al bajo nivel educativo de quienes manejan el producto en el campo, se generan la utilización indiscriminada de plaguicidas causando un incremento en el nivel de riesgo tanto para sí mismos como para los consumidores finales y el medio ambiente, la Comunidad Europea (CE), la Organización de Naciones Unidas para la Agricultura y la Alimentación (FAO), la Organización Mundial de la Salud (OMS) y algunos gobiernos han convertido el monitoreo y control de los pesticidas en actividades prioritarias para determinar la calidad y seguridad de los alimentos y han establecido límites máximos residuales (LMRs). (Ramirez \& Lacasaña, 2001). Así mismo, en nuestro país el diagnóstico, la vigilancia y el monitoreo de plaguicidas en alimentos aún no se ha implementado de forma eficaz, y existe una tendencia fuerte de los agricultores a usar plaguicidas en forma excesiva, debido especialmente a motivaciones económicas (Murcia \& Stashenko, 2008); (Arrieta y Quijano, 2016).

Por todo lo anterior, las entidades nacionales y regionales (Instituto 
Colombiano Agropecuario (ICA), Instituto Nacional de Vigilancia de Medicamentos y Alimentos (INVIMA), Entes Territoriales de Salud (ETS). Institutos Departamentales de Salud, entre otros) han intervenido con programas que mejoren las actividades agrícolas reduciendo los impactos generados por la producción de alimentos y con el fin de regular el uso indiscriminado de plaguicidas, creando normas que protejan los recursos naturales, evitando una mayor contaminación a los recursos ambientales y los efectos en la salud de los seres vivos cuando tienen contacto indirecto o indirecto con los plaguicidas. Lo anterior ha generado que se realicen estudios para detectar la presencia de estos compuestos en diversos alimentos, especialmente en frutas, dado que los métodos analíticos son costosos $y$ demorados y la industria requiere de métodos alternos que de manera indirecta pero en corto tiempo identifiquen dichos compuestos antes que el producto llegue al consumidor.

Cualquier metodología analítica para determinación de plaguicidas debe ser rápida, económica y aplicable, además con pocas modificaciones cuando se decida cambiar de matriz, en la actualidad la cromatografía de gases cumple con los requerimientos necesarios y confiables para determinar y cuantificar la presencia de trazas de plaguicidas en los productos mencionados, sin embargo, suele ser una técnica costosa, de difícil puesta a punto, mantenimiento y operación.

Plaguicidas. El concepto de plaguicidas es definido por la Food and Agriculture Organization (FAO) como «la sustancia o mezcla de ellas, destinada a prevenir, destruir o controlar plagas, incluyendo los vectores de enfermedad humana o animal; las especies no deseadas de plantas o animales que ocasionan un daño duradero u otras que interfieren con la producción, procesamiento, almacenamiento, transporte y comercialización de alimentos; los artículos agrícolas de consumo, la madera y sus productos, el forraje para animales o los productos que pueden administrárseles para el control de insectos, arácnidos $u$ otras plagas corporales». (Food Agriculture Organization United Nations (FAO), 2008).

Aunque los pesticidas son necesarios, los residuos de éstos en los alimentos y en el ambiente; provocan efectos negativos en la salud que dependen de la proporción con la que se absorbe el pesticida. Algunos efectos que se presentan son: toxicidad neurológica aguda, daño neurológico crónico, disfunción de los sistemas inmune, reproductivo y endocrino o cáncer (Dömötörová \& Matisová, 2008). Por lo que es necesario que su monitoreo y control se convierta en una actividad prioritaria para determinar la calidad y seguridad de los alimentos. Los plaguicidas se pueden clasificar de la siguiente manera: 


\section{Según el tipo de organismo que se} desea controlar: Insecticidas, acaricidas, fungicidas, herbicidas, nematicidas, molusquicidas, rodenticidas, avicidas.

\section{Según grupo químico del principio} activo: Compuestos organofosforados, compuestos carbamatos, compuestos organoclorados, piretroides, derivados del bipiridilo, triazinas, tiocarbamatos, derivados del ácido fenoxiacético, derivados de la cumarina, derivados del cloronitrofenol, compuestos organomercuriales, entre otros. A continuación se mencionan características generales de algunos pesticidas (Barbera C., 1989)

Tabla 1. Clasificación de los Plaguicidas según la Familia Química.

\begin{tabular}{|l|l|}
\hline \multicolumn{1}{|c|}{ Familia Química } & \multicolumn{1}{|c|}{ Ejemplos } \\
\hline Organoclorados & $\begin{array}{l}\text { DDT, aldrín, } \\
\text { endosulfán, endrín }\end{array}$ \\
\hline Organofosforados & $\begin{array}{l}\text { Bromophos, } \\
\text { diclorvos, malatión }\end{array}$ \\
\hline Carbamatos & $\begin{array}{l}\text { Carbaryl, methomyl, } \\
\text { propoxur }\end{array}$ \\
\hline Tiocarbamatos & $\begin{array}{l}\text { Ditiocarbamato, } \\
\text { mancozeb, maneb }\end{array}$ \\
\hline Piretroides & $\begin{array}{l}\text { Cypermetrin, } \\
\text { fenvalerato, } \\
\text { permetrín }\end{array}$ \\
\hline Derivados Bipiridilos & $\begin{array}{l}\text { Clormequat, diquat, } \\
\text { paraquat }\end{array}$ \\
\hline $\begin{array}{l}\text { Derivados del ácido } \\
\text { fenoxiacético }\end{array}$ & $\begin{array}{l}\text { Dicloroprop, piclram, } \\
\text { silvex }\end{array}$ \\
\hline $\begin{array}{l}\text { Derivados } \\
\text { cloronitrofenólicos }\end{array}$ & $\begin{array}{l}\text { DNOC, dinoterb, } \\
\text { dinocap }\end{array}$ \\
\hline $\begin{array}{l}\text { Derivados de } \\
\text { triazinas }\end{array}$ & $\begin{array}{l}\text { Atrazine, ametryn, } \\
\text { desmetryn, simazine }\end{array}$ \\
\hline $\begin{array}{l}\text { Compuestos } \\
\text { orgánicos del estaño }\end{array}$ & $\begin{array}{l}\text { Cyhexatin, dowco, } \\
\text { plictrán }\end{array}$ \\
\hline $\begin{array}{l}\text { Compuestos } \\
\text { inorgánicos }\end{array}$ & $\begin{array}{l}\text { Arsénico pentóxido, } \\
\text { obpa, fosfito de } \\
\text { magnesio, cloruro } \\
\text { de mercurio, }\end{array}$ \\
\hline
\end{tabular}

\begin{tabular}{|l|l|}
\hline & $\begin{array}{l}\text { arsenato de plomo, } \\
\text { bromuro de metilo, } \\
\text { antimonio, mercurio, } \\
\text { selenio, talio y } \\
\text { fósforo blanco }\end{array}$ \\
\hline $\begin{array}{l}\text { Compuestos de } \\
\text { origen botánico }\end{array}$ & $\begin{array}{l}\text { Rotenona, nicotina, } \\
\text { aceite de canola }\end{array}$ \\
\hline
\end{tabular}

Fuente: Adaptado y tomado de Ramírez \& Lacasaña, (2001)

\section{Según su persistencia al medio}

ambiente: Se clasifican en persistentes, poco persistentes, no persistentes (Tabla 2). Muchos plaguicidas persisten durante largos períodos de tiempo en el medio ambiente (hasta muchos años), como por ejemplo la vida prevista del $\mathrm{y}-\mathrm{HCH}$ en agua es de 191 días y la naturaleza persistente de los residuos de organoclorados en el medio ambiente plantean el problema de la toxicidad crónica para los animales y los seres humanos a través del aire, el agua y los alimentos de consumo (Kafilzadeh, 2015). De igual forma, el promedio de vida del $\gamma-\mathrm{HCH}$ y el $\alpha-\mathrm{HCH}$ en torno a la región de grandes lagos oscila alrededor de 3 a 4 años (Cortes \& Hites, 2000). Por lo tanto, el uso de un número de OCs, incluido el DDT, ha sido prohibido o restringido en el mundo debido a la alta persistencia y la bioacumulación en la naturaleza (OMS, 2003).

Se han detectado residuos de pesticidas organoclorados en ambientes costeros como se reportó en una investigación que se llevó a cabo en la Laguna Costera de Berre (Sureste de Francia), donde los sedimentos parecían estar 
significativamente

contaminados, especialmente con una concentración media de lindano de $0,05 \mathrm{mg} \mathrm{kg}-1$, que es $\leq$ cinco veces del umbral legal. (Arienzo, Masuccio, \& Ferrara, 2013). De igual forma, se han encontrado residuos en mariscos comúnmente consumidos por la población coreana, donde los bifenilos policlorados y el DDT fueron los contaminantes predominantes (Moon, Kim, Minkyu, Jun, \& Hee-Gu, 2009), en mamíferos marinos (Romanic, Klincic, Kljakovic-Gašpic, Kusak, Reljic, \& Huber, 2015), aves (Yohannes, Ikenaka, Nakayama, Mizukawa, \& Ishizuka, 2016) y humanos ( Thomas, et al., 2017). Además, presentan propiedades de transporte de largo alcance, como por lo que pueden ser llevados a largas distancias por medio de aire o del agua como es el caso del $\mathrm{HCH}$ y el Endosulfan que fueron transportados de tierras de cultivo a estuarios tropicales, lo que en última instancia provoca un grave riesgo para el Biota (Leadprathom, Parkpian, Satayavivad, Delaune, \& Jugsujinda, 2009). La gran persistencia de estos productos químicos en el medio ambiente y su fácil dispersión y desplazamiento en los cultivos ha sido un factor clave en el detrimento y deterioro de los ecosistemas y del aumento de enfermedades y patologías relacionadas con humanos y otras especies de la biosfera (Albert \& Rendon-Von Osten, J., 1988).
Tabla 2. Clasificación de los plaguicidas según su vida media de efectividad

\begin{tabular}{|l|l|l|}
\hline Persistencia $^{\text {a }}$ & Vida media $^{\text {b }}$ & $\begin{array}{l}\text { Ejemplo } \\
\text { s }\end{array}$ \\
\hline No persistente & $\begin{array}{l}\text { De días } \\
\text { hasta 12 } \\
\text { semanas }\end{array}$ & $\begin{array}{l}\text { Malatión, } \\
\text { diazinón, } \\
\text { carbarilo, } \\
\text { diametrín }\end{array}$ \\
\hline $\begin{array}{l}\text { Moderadament } \\
\text { e persistente }\end{array}$ & $\begin{array}{l}\text { De 1 a 18 } \\
\text { meses }\end{array}$ & $\begin{array}{l}\text { Paratión, } \\
\text { lannate }\end{array}$ \\
\hline Persistente & $\begin{array}{l}\text { De varios } \\
\text { meses a 20 } \\
\text { años }\end{array}$ & $\begin{array}{l}\text { DDT, } \\
\text { aldrín, } \\
\text { dieldrín }\end{array}$ \\
\hline Permanentes & $\begin{array}{l}\text { Indefinidame } \\
\text { nte }\end{array}$ & $\begin{array}{l}\text { Producto } \\
\text { s hechos } \\
\text { a partir } \\
\text { de } \\
\text { mercurio, } \\
\text { plomo, } \\
\text { arsénico }\end{array}$ \\
\hline
\end{tabular}

Fuente: Adaptado y tomado de Ramírez \& Lacasaña, (2001). ${ }^{a}$ Capacidad de una sustancia o un compuesto, de permanecer en un sustrato del ambiente en particular, después de que ha cumplido el objetivo por el cual se aplicó. b Lapso de tiempo necesario para que se degrade la mitad del compuesto o mezcla aplicada.

Según su toxicidad aguda (O.M.S.): Esta se basa principalmente en la toxicidad por vía oral en ratas y ratones. Usualmente la dosis se registra como el valor DL50 (Dosis Letal Media) que es la dosis requerida para matar al $50 \%$ de la población de animales de prueba y se expresa en términos de $\mathrm{mg} / \mathrm{kg}$ del peso del cuerpo del animal.

Tabla 3. Clasificación de los plaguicidas según su toxicidad, expresada en DL50 (mg/kg)

\begin{tabular}{|l|l|l|}
\hline $\begin{array}{l}\text { Clas } \\
\text { e }\end{array}$ & Toxicidad & Ejemplos \\
\hline
\end{tabular}




\begin{tabular}{|l|l|l|}
\hline $\begin{array}{l}\text { Clase } \\
\text { IA }\end{array}$ & $\begin{array}{l}\text { Extremadament } \\
\text { e peligrosos }\end{array}$ & $\begin{array}{l}\text { Paratión, } \\
\text { dieldrín }\end{array}$ \\
\hline $\begin{array}{l}\text { Clase } \\
\text { IB }\end{array}$ & $\begin{array}{l}\text { Altamente } \\
\text { peligrosos }\end{array}$ & $\begin{array}{l}\text { Eldrín, } \\
\text { diclorvs }\end{array}$ \\
\hline $\begin{array}{l}\text { Clase } \\
\text { II }\end{array}$ & $\begin{array}{l}\text { Moderadamente } \\
\text { peligrosos }\end{array}$ & $\begin{array}{l}\text { DDT,clordan } \\
\text { o }\end{array}$ \\
\hline $\begin{array}{l}\text { Clase } \\
\text { III }\end{array}$ & $\begin{array}{l}\text { Ligeramente } \\
\text { peligrosos }\end{array}$ & Malarión \\
\hline
\end{tabular}

Fuente: Adaptado y tomado de Ramírez \& Lacasaña, (2001)

Los plaguicidas son insumos esenciales utilizados en la agricultura moderna para controlar las plagas y enfermedades e incrementar la productividad de los cultivos disminuyendo los riesgos y pérdidas de los sistemas agrícolas. El uso generalizado de tales productos se debe a las propiedades biocidas y selectividad que poseen; por ello, constituyen el método habitual de lucha contra las plagas. (Pérez, Navarro, \& Miranda, 2013). Durante la década de los 30 y hasta nuestros días son muy utilizados en la industria agrícola a nivel mundial y han tenido notables consecuencias nocivas de carácter mutagénico y canceroso (Pabuena, et al., 2015) debido a su persistencia y residualidad que se acumula en tejidos humanos (Thomas, et al., 2017), por lo que surge la necesidad de investigar el uso de dichas sustancias y sus repercusiones en los consumidores.

Estas sustancias químicas se utilizan en frutas, verduras, trigo, arroz, aceitunas y canola destinada para aceite y en cultivos no alimentarios, tales como algodón, césped y flores. La alta demanda en el uso de plaguicidas genera consecuencias que afectan los ecosistemas ocasionando desequilibrios debido a la residualidad que se genera en los alimentos (Bempah, Agyekum, Akuamoa, Frimpong, \& BuahKwofie, 2016; Castro, 2005). Además, la manipulación y aplicación de plaguicidas puede ocasionar riesgos para el ser humano, ya sea por ser usuario o consumidor de vegetales, frutas $y$ productos tratados, así como afectar el propio cultivo y su entorno, el ganado, la fauna terrestre y acuícola, y en definitiva el equilibrio ecológico dejando residuos de plaguicidas en el suelo durante años. (Barron, y otros, 2017).

El uso dado a los plaguicidas ha sido múltiple y variado, esto es debido a que las tierras de cultivo del mundo son limitadas y la población está creciendo constantemente, lo que conlleva a realizar todos los esfuerzos para aumentar la producción de cultivos con el fin de garantizar la seguridad alimentaria (Zhang, 2008). De igual forma, sin el uso de plaguicidas, el rendimiento y la calidad de los cultivos pueden ser severamente reducidos por malas hierbas, plagas $y$ enfermedades, lo cual ocasionaría pérdidas de más del $50 \%$ de los cultivos vegetales por daño de plagas (Wang, Wang, Huo, \& Zhu, 2015), lo que indica que los plaguicidas son, por lo tanto, indispensables en la producción agrícola 
moderna. Actualmente, hay más de 865 plaguicidas registrados en todo el mundo (Wanwimolruk, Kanchanamayoon, Phopin, \& Prachayasittikul, 2015). Ahora bien, el hecho que los compuestos químicos reduzcan los daños ocasionados por la plaga en los cultivos, no justifica que se utilicen indiscrimidamente, sin regirse por la normativa vigente y sopresando los límites máximo permitidos, ocasionando consecuencias residuales en los alimentos, si no en el consumidor, en la fauna y la flora de todo el entorno. En la tabla 4 se muestra la diversidad de aplicaciones que tienen los pesticidas

Tabla 4. Usos más frecuentes de los Plaguicida.

\begin{tabular}{|c|c|}
\hline Actividad & $\begin{array}{l}\text { Uso } \\
\end{array}$ \\
\hline Agricultura & $\begin{array}{l}\text { Control de las múltiples plagas } \\
\text { que afectan las cosechas en } \\
\text { cualquiera de sus etapas. }\end{array}$ \\
\hline & $\begin{array}{l}\text { Control de vectores de } \\
\text { enfermedades como malaria, } \\
\text { dengue, enfermedad de } \\
\text { Chagas, oncocercosis, peste, } \\
\text { fiebre amarilla, filariasis, } \\
\text { tripanosomiasis, } \\
\text { esquistosomiasis, } \\
\text { leishmaniasis y tifo. } \\
\text { Control de plagas (roedores) y } \\
\text { erradicación de plantaciones } \\
\text { cuyo producto final sea droga } \\
\text { ilícita. }\end{array}$ \\
\hline $\begin{array}{l}\text { Ganadería y } \\
\text { cuidado de } \\
\text { animales } \\
\text { domésticos }\end{array}$ & $\begin{array}{l}\text { En la desinfección de ganado } \\
\text { ovino y de animales } \\
\text { domésticos como perros y } \\
\text { gatos. }\end{array}$ \\
\hline $\begin{array}{l}\text { Tratamiento } \\
\text { de } \\
\text { estructuras }\end{array}$ & $\begin{array}{l}\text { Tratamiento de edificios } \\
\text { públicos y privados, oficinas, } \\
\text { hospitales, hoteles, cines, } \\
\text { teatros, restaurantes, } \\
\text { escuelas, supermercados, } \\
\text { tiendas de departamentos, } \\
\text { instalaciones deportivas, } \\
\text { bodegas de almacenamiento } \\
\text { de alimentos y en la industria } \\
\text { ferroviaria y de navegación } \\
\text { marítima y aérea. }\end{array}$ \\
\hline $\begin{array}{l}\text { Mantenimien } \\
\text { to de }\end{array}$ & $\begin{array}{l}\text { Tratamiento de parques, } \\
\text { jardines, áreas de recreo, }\end{array}$ \\
\hline
\end{tabular}

\begin{tabular}{|c|c|}
\hline áreas verdes & $\begin{array}{l}\text { campos de golf y autopistas, } \\
\text { vías férreas, andenes, torres } \\
\text { con líneas de alta tensión y } \\
\text { postes. }\end{array}$ \\
\hline $\begin{array}{l}\text { Mantenimien } \\
\text { to de } \\
\text { reservas de } \\
\text { agua }\end{array}$ & $\begin{array}{l}\text { Tratamiento de grandes } \\
\text { reservas de agua, naturales o } \\
\text { artificiales, presas, embalses, } \\
\text { diques, depósitos, estanques } \\
\text { piscícolas, canales, albercas y } \\
\text { piscinas }\end{array}$ \\
\hline Industria & 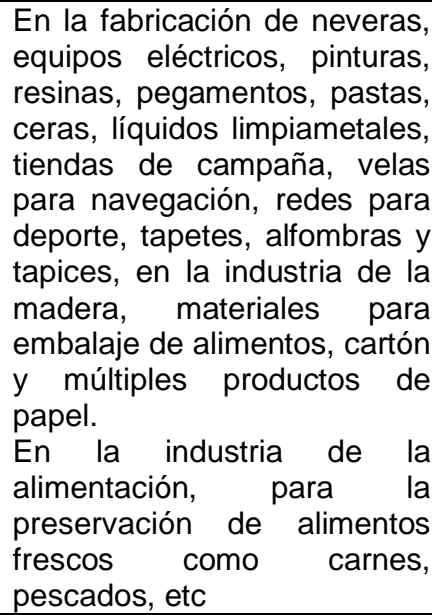 \\
\hline Hogar & $\begin{array}{l}\text { Incorporados en productos } \\
\text { como cosméticos, champús, } \\
\text { jabones y repelentes de } \\
\text { insectos. Se usan en el lavado } \\
\text { y secado de alfombras, en } \\
\text { desinfectantes caseros y en } \\
\text { productos para el cuidado de } \\
\text { mascotas y plantas, además } \\
\text { del uso de insecticidas }\end{array}$ \\
\hline
\end{tabular}

Fuente: Adaptado y tomado de Ramírez \& Lacasaña, 2001; Moses, (1993).

El tema de los residuos de plaguicidas en los alimentos representa un tema polémico y complicado de interés para los consumidores, productores y procesadores de alimentos, reguladores, legisladores y científicos de todo el mundo. Los niveles permisibles de residuos de plaguicidas en los alimentos, conocidos como tolerancias en los Estados Unidos y como límites máximos de residuos (LMR) en gran parte del mundo, se perciben ampliamente y de manera inapropiada como niveles de preocupación por la seguridad. (Codex 
Alimentarius, 1996). Por tal motivo y con el objetivo de proteger la salud de los consumidores y asegurar que se aplique al alimento solamente la cantidad mínima de plaguicida que se necesita, la legislación Colombiana sigue los Límites Máximos de Residuos (LMR's). El sector agrícola es el que más emplea compuestos químicos como pesticidas para mitigar la proliferación de plaga que merman la calidad y cantidad de las cosechas de alimentos; realizando un consumo del $85 \%$ de la producción mundial de este producto. Su empleo indiscriminado, dependiendo de la toxicidad, el tiempo y el tipo de exposición se ha convertido en una problemática a nivel del país ya que ha dificultado el acceso a mercados internacionales, donde las normas son altamente restrictivas con respecto a la presencia de trazas de organoclorados, organofosforados y carbamatos en alimentos frescos 0 procesados. La preocupación de los consumidores con respecto a los residuos de plaguicidas ha dado lugar, en parte, al crecimiento sostenido de la industria de alimentos orgánicos, poniendo en tela de juicio el escrutinio reglamentario de las deficiencias percibidas en el muestreo de alimentos y en los métodos analíticos utilizados. (Winter \& Jara, 2015).

El empleo de los plaguicidas como productos químicos para el control de plagas se hace conforme a las normas de inocuidad establecidas por los entes de control reglamentando límites máximos permisibles de residuos para que el riesgo a corto y mediano plazo en los consumidores sea mínimo. Sin embargo, en ocasiones los límites son rebasados, o bien, se tiene la presencia de varios residuos en un mismo producto, y pese a que las concentraciones estén por debajo de los valores aceptables, el riesgo potencial se incrementa al considerarse un efecto aditivo y perjudicial para la salud, como es el caso de la fresa, la cual es considerada una de las frutas con mayor contenido de plaguicidas y se encuentra dentro de lista de alimentos clasificados como "docena sucia" por la Environmental Working Group (EWG). (Winter \& Jara, Pesticide food safety standards as companions to tolerances and maximum residue limits, 2015).

Esta investigación se centra en el estudio de los pesticidas Organoclorados, los cuales al ser compuestos orgánicos, tienden a reducir su reactividad. Esta estabilidad se manifiesta como una persistencia en el medio ambiente, que se incrementa a cloración del compuesto, por ejemplo; el diclorobenceno no es demasiado persistente, pero sí lo es mucho más el hexaclorobenceno. El gran tamaño y masa del átomo de cloro resulta en una gran molécula que tiene una presión de vapor muy reducida, incrementa el punto de ebullición y reduce la solubilidad en 
agua (Garrido, 1988 en García Cambero, 2002). La adición de átomos de cloro incrementa así la partición de los lípidos y por consiguiente, la bioconcentración, al igual que la liposolubilidad y la estabilidad de estos compuestos facilitan su acumulación en los tejidos grasos, asimismo, una propiedad común de los derivados Ciclodiénicos, es su gran persistencia en el medio ambiente y de modo especial su identidad de comportamiento biológico al ser transformados en sus epóxidos; así el aldrín pasa a dieldrín y el heptacloro a heptacloro epóxido tanto en el suelo como en animales o en sistemas biológicos. $\mathrm{Si}$ bien se puede rastrear el empleo de substancias para proteger las cosechas desde la antigüedad, el concepto moderno de plaguicida surge en el siglo XIX en que se sintetizaron múltiples substancias cuyas propiedades tóxicas e insecticidas se descubrieron y utilizaron más adelante. La investigación de compuestos arsenicales dio lugar al empleo del arsenito de cobre para combatir un tipo de escarabajo en EE.UU., y la extensión de su empleo promovió la primera legislación conocida sobre pesticidas en el año 1900. El diclorodifeniltricloroetano

(DDT), sintetizado en 1874, demostró su potencia insecticida en 1939 y comenzó a ser utilizado como tal en 1942. El hexaclorocicloexano $(\mathrm{HCH})$, sintetizado en 1825, se usó como gas de guerra en la $1^{\text {a }}$ Guerra Mundial y como insecticida en
1942. A partir de la $2^{a}$ mitad del siglo XX se acelera la síntesis de productos organofosforados (dimefox (1949), malation (1950) y de carbamatos (carbaryl (1956), aldicarb (1965), Costa, Galli, \& Murphy, (1986) en Ferrer, 2003).

\section{Métodos e investigaciones para determinación de pesticidas} organoclorados. Debido a su amplio uso, estabilidad, toxicidad selectiva y bioacumulación, los plaguicidas están entre las sustancias más tóxicas que contaminan el medio ambiente. Son particularmente peligrosos en las frutas y hortalizas, por las cuales las personas están expuestas a ellos. Por lo tanto, es fundamental vigilar los residuos de plaguicidas en las frutas y hortalizas utilizando todos los métodos analíticos disponibles (Fenik, ankiewicz, \& Biziuk, 2011).

Recientemente, en la literatura se ha reportado diferentes métodos modernos en la extracción de los plaguicidas de matrices de frutas y vegetales, como las hortalizas, se han empleado las técnicas de extracción como: extracción en fase sólida, SPE (Ambrus, et al., 2005; Carabias-Martínez, Rodríguez-Gonzalo, Miranda-Cruz, Domínguez-Álvarez, \& HernándezMéndez, 2007; X. Yang, et al., 2011; Zhao, et al., 2016); microextracción en fase sólida SPME (Chai \& Tan, 2009; Hirahara, et al., 2005; Li, Wang, Yan, Li, \& Pan, 2016), extracción en fase sólida dispersa, SPDE 
(Escalona, Galarraga, Fernández, Centeno, Velásquez, \& Pérez, 2012; Farajzadeh, Yadeghari, \& Khoshmaram, 2017; Liu, Kong, Gong, Miao, Qi, \& Yang, 2015); extracción con disolventes, LLE (Banerjee, et al., 2012; Duodua, Goonetillekeb, \& Ayoko, 2016; Escuderos M., Santos D., Rubio B., \& Polo D., 2003; Musaiger , Al-Jedah, \& D' Zousa, 2008;); extracción con fluidos súper crítico SFE (Mohamed, EL-Saeid, Saleh, \& AL-Dosari, 2010; Poustka, Holadová, \& Hajšová , 2003; Rissato, Galhiani, \& Gerenutti, 2005; Velasco, Villada, \& Carrera, 2007); extracción asistida con ultrasonidos, AUE (Rodríguez-Gonzalo, Carabias-Martínez, Miranda-Cruz, Domínguez-Alvarez, \& Hernández-Méndez, 2009); extracción asistida con microondas, MAE y SPE (Farajzadeh, Asghari, \& Feriduni, 2016; Fuentes E, Báez \& Díaz, 2009; Wang, Ding, \& Ren, 2016); extracción con membranas permeables-MPE (van Pinxteren, Bauer, \& Popp, 2009) y extracción acelerada con disolventes, ASE ((Cervera, et al., 2010; Mijangros R., 2010), los cuales reducen el tiempo de análisis, permiten poca manipulación de la muestra y minimizan el volumen del solvente. (TrticPetrovic,

Dordevic,

\begin{tabular}{|c|c|c|c|c|c|c|}
\hline Caltines & $\begin{array}{l}\text { Plagiididas } \\
\text { andizados }\end{array}$ & $\begin{array}{c}\text { Metadodo } \\
\text { preparcition } \\
\text { Etrración }\end{array}$ & $\begin{array}{c}\text { Limpizal } \\
\text { concentrabina de be } \\
\text { mustra }\end{array}$ & Solvents utlinados & Detectiono Analifica & Atator \\
\hline Hendilins (1)" & 5 & MSPD & 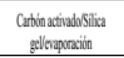 & Didornatano & GCEED & $\begin{array}{c}\text { Allasantal. } \\
2001 \\
\text { 201. }\end{array}$ \\
\hline Fnatsil(i) & 7 & Moringh, filtrain & UE, craporasion & Acethas & GCMS & Allana 2008 \\
\hline Hordinus (3) & 3 & 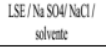 & 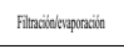 & Anetato det tho & HPLC & Buigetal. 2009 \\
\hline $\begin{array}{l}\text { Fntasy Hortings } \\
(400)\end{array}$ & 14 & ISSPD & DSPFflomenganizaicin & Aetata de te the & GC.MS & $\begin{array}{l}\text { Bemph, Agydum } \\
\text { Akamo, Fimpone, } \\
\text { \& Bubl-Kuofic, 2016 }\end{array}$ \\
\hline $\begin{array}{l}\text { Hutalins (3) } \\
\text { Frtus (1) } \\
\text { Caralss(3) }\end{array}$ & 118 & 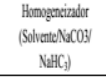 & 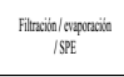 & Antatab de tetio & TLC & Ambros atal 2005 \\
\hline 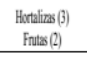 & 399 & QUECLERSE & DSPE & Actatato de tillo & GC-ESWHS IS & Bancipictal 2012 \\
\hline Hardinas (4) & 9 & 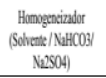 & SPElaparacion & Actatrode teilo & GCEED & Bemphintal 2012 \\
\hline $\begin{array}{l}\text { Hixtalins (6) } \\
\text { Fintos (3) }\end{array}$ & 121 & $\begin{array}{l}\text { QuElenERSE } \\
\text { Modificido }\end{array}$ & Sohterir + Meald de sals & Actantitilo & GC-NSWS (QQP) & Camino-Sindie 2011 \\
\hline $\begin{array}{l}\text { Hentilins (l) } \\
\text { Fintas (2) }\end{array}$ & 130 & ASE & ASElacio N2/GPC & Anetato de thilo & $\begin{array}{c}\text { GC-NISMS } \\
(0 \times 0)\end{array}$ & Centeared. 2010 \\
\hline $\begin{array}{l}\text { Hetalinas (5), } \\
\text { Fintas (1) } \\
\text { Cerals (1) } \\
\end{array}$ & 6 & PLE & SPElemporacioio $\mathrm{N}_{2}$ & 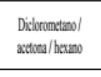 & HPLCNACE & $\begin{array}{c}\text { Canblias-latitice et } \\
\text { al. } 2007\end{array}$ \\
\hline Hontlizs (?) & 15 & MSPE & $\begin{array}{l}\text { SPE milicapu } \\
\text { CBB/PSA }\end{array}$ & 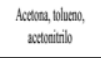 & CCEED & 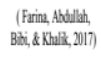 \\
\hline $\begin{array}{l}\text { Hertalins (4) } \\
\text { Fintas (5) }\end{array}$ & 239 & 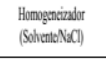 & SPEluapraxion & Actennitibibo $+\mathrm{N}_{\mathrm{S}} \mathrm{Cl}$ & GCMS & Fillinan ed a.2000 \\
\hline $\begin{array}{l}\text { Hertilins (6) } \\
\text { Fintas (2) }\end{array}$ & 31 & 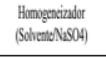 & Filtraidereaporacion & Actuta de teilo & LC.ESH-1S.-1S & Caridioe ed d.2004 \\
\hline
\end{tabular}

Dujakovic, Kumric, Kavasiljevic, \& Lausevic, 2010 en Pérez, Salas, \& Amaro, 2015).

Para desarrollar correctamente estas metodologías se debe tener presente las características de las muestras objeto estudio y las propiedades fisicoquímicas de los plaguicidas en contacto con la matriz, así como en el proceso de extracción y análisis instrumental. Es esencial emplear las técnicas y metodologías que permitan obtener resultados reproducibles, confiables y puedan emplearse en análisis de rutina, esto implica utilizar técnicas de extracción adecuadas y establecer las condiciones apropiadas de operación de los equipos disponibles. Debido a la gran problemática que ha surgido con la utilización de pesticidas y a la importancia de determinar la presencia de estos en muestras de alimentos, ambientales y 
biológicas, algunas instituciones gubernamentales han diseñado una serie de métodos analíticos para la determinación de plaguicidas que permitan cuantificar concentraciones iguales $O$ inferiores a los valores de LMR establecidos para cada uno de los diferentes contaminantes presentes en la matriz objeto estudio (Tabla 5).

Tabla 5. Técnicas de extracción, Limpieza y detección de Plaguicidas en hortalizas, frutas y cereales.

\begin{tabular}{|c|c|c|c|c|c|c|}
\hline Calling & Plageidides anlandos & 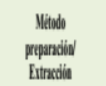 & $\begin{array}{l}\text { Limpinal } \\
\text { curentrocinde } \\
\text { busotn }\end{array}$ & Shatto ullindos & $\begin{array}{l}\text { Deteribin } \\
\text { falitita }\end{array}$ & Aatur \\
\hline Howilize(2) & 9 & Honyganiablir & SPE & 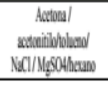 & ac & 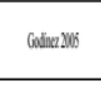 \\
\hline Hoting (3") & 3 & LSE & SEFlinanación & Aetercitith & cerms & 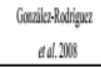 \\
\hline 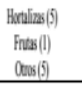 & 160 & Abluadar. LSE & FiltaüüNicio MSPE & Actuto de tilo & $\begin{array}{c}\text { GC.PPD, } \\
\text { GC:PD, CCEECD }\end{array}$ & Hrahus ad .X05 \\
\hline $\begin{array}{l}\text { Handins(l) } \\
\text { hatse(18) }\end{array}$ & 30 & LEE,QEEABSS & CP,SPE, USPD & 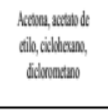 & $\begin{array}{l}\text { GCEED, GC.NPD, } \\
\text { GC.ID, GC.NSy } \\
\text { GC.USWS; } \\
\text { LC.NSMS }\end{array}$ & Hyinh ad.2011 \\
\hline Bralins(1) & 12 & $\begin{array}{l}\text { LSE wan Agitrie, } \\
\text { щF }\end{array}$ & 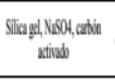 & 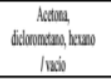 & 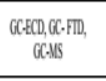 & $\begin{array}{l}\text { Mndaly } \\
\text { Singlavilo }\end{array}$ \\
\hline $\begin{array}{l}\text { Howlins (12) } \\
\text { Fats (18) }\end{array}$ & 57 & LSENESOA, Fithaion & 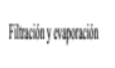 & Antas de the & LCASWS & 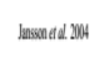 \\
\hline 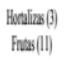 & $\theta$ & QUEChERS & 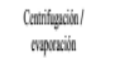 & Alatsiatib & LCEESIS & Kanlizata 2000 \\
\hline $\begin{array}{l}\operatorname{Hrdins}(\text { (t) } \\
\text { Fins (f) }\end{array}$ & 25 & QEECHESS & SPElowaravia & Adatinitib & $\begin{array}{l}\text { GCUSLLLC. } \\
\text { USWS }\end{array}$ & 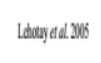 \\
\hline Fatal(l) & 19 & Finsaion & GCPhroyganizina & Actuto de cilo & GCAR,EEONPD & 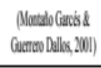 \\
\hline 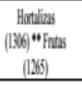 & 74 & LSE-Agigaia & 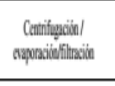 & Actato de tallo & LCEESHSUS & Oadili ad:204 \\
\hline $\begin{array}{l}\text { Honings(3) } \\
\text { Frase(2) }\end{array}$ & 2 & SIE & SFE & $\omega_{1}$ & $G C . N D, G C E E D$ & Pussue al 2003 \\
\hline
\end{tabular}

Nota: * El valor se refiere al número de cultivos estudiados; ${ }^{* *}$ el valor se refiere al número de muestras analizadas de cada grupo de cultivos.

Método Quechers. (Quick, Easy, Cheap, Effective, Rugged and Safe), siendo este según sus siglas; rápido, fácil, económico, efectivo, robusto y seguro para la extracción de una amplia gama de plaguicidas en matrices de frutas $y$ verduras con un alto porcentaje de humedad. Este nuevo método de preparación de muestras surgió de la necesidad de desarrollar métodos multiresiduos rápidos y económicos que proporcionen resultados de gran calidad y fiabilidad (Rejczak \& Tuzimski, 2017; Socas-Rodríguez, González-Sálamo, Herrera-Herrera, Hernández-Borges, \& Rodríguez-Delgado, 2017). Es un método muy aplicado a nivel mundial para la determinación de residuos de pesticidas en alimentos, debido a las múltiples ventajas que presenta ya que a través de un procedimiento sencillo, las muestras se preparan para el análisis de las muestras mediante GC, GC/MS o LC, LC/MS. QuEChERS, utiliza menor cantidad de muestra, en menos de 30 minutos, el analista es capaz de realizar dos o más extracciones al tiempo, no se requiere gran cantidad de material de vidrio y la cantidad de pasos de extracción se disminuye. Un amplio espectro de plaguicidas ha sido analizado usando QuEChERS, incluyendo plaguicidas no-polares, polares y planares (Anastassiades, Lehotay, Stajnbaher, \& Schenck, 2003; AOAC Official Method, 2007; Páez \& Martínez, 2015), ocupando una posición relevante en el análisis de residuos de plaguicidas. Las características inicialmente propuestas 
para nombrar el método (rápido, fácil, barato, eficaz, robusto y seguro) proporcionan numerosos beneficios desde un punto de vista analítico, y este aspecto ha llevado a su aplicación en la mayoría de los laboratorios oficiales para el análisis de este tipo de residuos. De igual forma, se han determinado efectivamente otros grupos diferentes de compuestos con especial interés en los campos medioambiental, alimentario y biológico en una amplia variedad de matrices complejas.

En términos generales, la metodología QuEChERS ha sido validada para una gran cantidad de frutas y vegetales de diferentes características, mostrando resultados bastante favorables para ser utilizados en el análisis de plaguicidas en laboratorios de rutina, lo que lo hace un método atractivo para su implementación. A partir del método original se han desarrollado diversas modificaciones basadas en el uso de disoluciones tamponadas para mejorar la eficacia de la extracción de compuestos dependientes del $\mathrm{pH}$, tal es el caso del método 2007.01 (AOAC 2007) en el cual se utiliza acetonitrilo con un $1 \%$ en ácido acético para formar un tampón con acetato de sodio, lográndose obtener un $\mathrm{pH}$ alrededor de 4,5 y se elimina la adición de $\mathrm{NaCl}$. Otra modifcación del método QuEChERS fue realizada por Anastassiades (2003) y se validó por la European Committee for standardization
(CEN) como el método estándar EN 15662, en donde se utilizó buffers citrato (BS EN 15662:2008 2008).

\section{Técnicas cromatográficas. La} determinación analítica de plaguicidas en frutas, vegetales $y$ hortalizas ha sido revisada por varios autores, quienes indican que en la mayoría se han empleado las técnicas cromatográfcas acopladas a un sistema de detección y/o analizador, y con menor frecuencia las técnicas electroquímicas, inmunoensayos, electrocromatografía, biosensores de iones y electroforesis capilar (Ahmed 2001, Lambropoulou y Albanis 2007; Soler y Picó 2007; Sharma et al., 2010; Fenik et al., 2011). Pérez, Navarro y Miranda (2013) realizaron una revisión bibliográfica de las técnicas cromatográficas utilizadas en el análisis de plaguicidas en frutas $y$ vegetales (incluidas hortalizas), y con base a diferentes publicaciones científicas recientes, describen que las técnicas más empleadas son: cromatografía de gasesespectrometría de masas (Fillion, Sauve \& Selwyn,. 2000; Guerrero \& Herrera, 2016; Lehotay, 2005). En 2010 Norli, Christiansen, \& Holen, realizaron una evaluación independiente de un programa de reporte por deconvolución Deconvolution Reporting Software (DRS, por sus siglas en inglés) que emplea AMDIS como núcleo para el análisis de residuos de plaguicidas poniendo a prueba la capacidad para detectar plaguicidas en 
el rango de 20 a $100 \mu \mathrm{g} / \mathrm{kg}$ en frutas y hortalizas. (Philip, Wylie, \& Meng, 2004). El estudio mostró resultados aceptables teniendo en cuenta las frecuentes dificultades al analizar plaguicidas volátiles "problemáticos". Conforme a los resultados encontrados por AMDIS, los investigadores definieron un Límite de Identificación (LI) el cual aseguraba una relación señal-ruido $(\mathrm{S} / \mathrm{N})$ mayor que 10. Cabe mencionar que en este estudio se empleó el método QUEChERS con el objetivo de evitar las interferencias de matriz y mantener altos porcentajes de recuperación (más del $95 \%$ de los compuestos entre 80 - $110 \%$ ). Un ensayo posterior de identificación mostró que algunos compuestos problemáticos tenían puntajes de comparación entre el 70 - $90 \%$ de coincidencia, aunque sólo el $3 \%$ del total de los puntajes para las determinaciones estuvieron por debajo del criterio $70 \%$. Aun así, es importante aclarar que la sensibilidad baja es el principal obstáculo para este tipo de técnicas automatizadas en el análisis de residuos que emplean modo scan, puesto que sin importar qué tan bien se haya purificado un extracto, el perfil de la matriz siempre tendrá una influencia significativa en las intensidades de los iones del analito, y en estas condiciones el método de comparación podría fallar por contaminación del espectro del analito con compuestos endógenos coeluyentes en una separación incompleta. cromatografía de gases-espectrometría de masas en tándem, GC-MS/ MS (Camino-Sánchez et al., 2011, Hjorth et al., 2011); cromatografía de gases - espectrometría de masas con cuadrupolo de ionización de triple impacto de electrones, GC-EI-MS/MS (Banerjee et al., 2012); cromatografía de gasesespectrometría de masas de trampa de iones, GC-MS-TI (Hjorth et al., 2011); cromatografía de gases-espectrometría de masas de tiempo de vuelo, GC-MS TOF (Ferrer I. \& Zweigmenbaum, 2011); cromatografía de gases con detector NPD (Escuderos-Morenas et al., 2003, Hjorth et al. 2011); cromatografía de líquidos de alta precisión, HPLC (Baig et al., 2009); cromatografía de gases-espectrometría de masas de ionización térmica, GC-TIMS (González-Rodríguez et al., 2008); cromatografía líquida-espectrometría de masas de ionización electrospray (Garrido et al. 2004); cromatografía líquidaespectrometría de masas en tándem (Garrido et al., 2004, Jansson 2004, Lehotay et al., 2005, Kmellár et al., 2008, Hjorth et al., 2011), cromatografía de gases con detector de captura de electrones y/o detector fotométrico de flama, GC-ECD y GC FPD (Bempah, Buah-Kwofie, Enimil, Blewub \& Agyei-Martey, 2012; Hirahara et al., 2005, Musaiger, Al-Jedah \& D' Zousa, 2008, Mandal y Singh 2010; Sapahin, Makahleh, \& Saad, 2015; Yu-feng, Al-Jedah \& D' Zousa, 2011); donde Rissato, Galhiane, \& Gerenutti (2005) presentaron un método rápido y seguro para determinar 25 compuestos seleccionados de cuatro 
clases de pesticidas, teniendo como muestras: manzanas, uvas, peras y fresas frescas, de supermercados locales. Utilizaron dos métodos de extracción: extracción sólido-líquido, usando acetona, y extracción con fluido supercrítico. La limpieza de las muestras fue realizada con cartuchos de extracción de aminopropil seguido por análisis de cromatografía de gases acoplada con un equipo de detección de captura de electrones. La confirmación de los pesticidas fue realizada con un detector de espectrometría de masa usando un método selectivo de monitoreo de iones. La recuperación de la mayoría de pesticidas en las muestras fortificadas fue a un nivel de 0.04-0.10 mg/kg, fue de 77-96 $\%$ para ambos métodos. El método de extracción con fluido supercrítico presentó buen límite de detección (menor que 0.01 $\mathrm{mg} / \mathrm{kg}$, dependiendo del pesticida y de la matriz), lo que permitió concluir que el método minimizó los daños ambientales, tiempo y el trabajo de laboratorio.

Sistema multisensorial. Ortiz S. (2016) describe en su trabajo de investigación que un sistema multisensorial está basado en sensores, los cuales pueden intentar imitar el comportamiento de los sentidos en el ser humano, el olfato y el gusto. La investigación con sistemas multisensoriales data ya de aproximadamente 30 años y durante los años 80 los investigadores se centraron en una máquina con características olfativas que sería llamada nariz electrónica y que constaría de una gran variedad de sensores. En la actualidad, los sistemas multisensoriales son comparados constantemente con equipos de cromatografía o espectrometría para corroborar que los resultados obtenidos (data) sean confiables. Los datos obtenidos pueden ser analizados por diferentes técnicas: análisis de componentes principales (PCA), PLS, análisis de discriminación funcional, análisis de clúster, lógica difusa y redes neuronales.

Nariz Electrónica (NE). Se define como un sistema de olfato electrónico utilizado como "instrumento que comprende una matriz de sensores químicos con sensibilidades solapadas y un avanzado sistema de reconocimiento de patrones capaces de identificar aromas simples y/o complejos" (Gualdron, 2006). El objetivo de este equipo es analizar cualitativamente aromas difíciles de percibir, reconociendo sus huellas olfativas, valorando los componentes de la muestra a analizar en todo su conjunto y no de una manera cuantitativa. (Lozano, Arroyo, Santos, Cabellos, \& Horrillo, 2008). El propósito general del sistema de sensores de una nariz electrónica es el de emular el sistema olfativo diferenciando adecuadamente los distintos tipos de volátiles que le sean requeridos, para la empresa alimentaría el uso de estos sistemas cada vez es mayor y está más en auge especialmente en el 
análisis sensorial de sustancias complejas. (Araque O., 2014).

Persaud y Dodd (1982), orientaron sus estudios en entender los procesos del olfato biológico, utilizando un conjunto de sensores semiconductores de óxidos metálicos, y luego ampliaron su investigación a sensores basados en polímeros conductores, mientras que, el otro grupo propuso un aparato para detectar, identificar y medir una amplia variedad de productos químicos y mezclas transportadas por ferrocarriles, barcos y camiones. Actualmente en la industria de alimentos se han realizado múltiples investigaciones con la utilización de la Nariz Electrónica para la clasificación de aromas y olores en diferentes productos, de ahí que el campo de aplicación se ha extendido al análisis en medio ambiente, medicina, seguridad y agroindustria.

En términos generales la nariz electrónica es un dispositivo compuesto por cuatro etapas funcionales: concentración y captura de los volátiles, electrónica de control, y procesamiento de la información. (Gualdron, Duran, Ortiz, \& Araque, 2014).

Un sistema de olfato electrónico estándar está formado por tres elementos principales: un sistema de medición, compuesto por una técnica de extracción de aroma o sistema de flujo de aire, que transporta los compuestos volátiles de las muestras hasta un arreglo de sensores químicos que transforman la información recibida en señales eléctricas; un sistema de extracción de los rasgos característicos de la muestra analizada, mediante la cuantificación y transformación en datos de las señales de los diferentes sensores y un sistema de reconocimiento de patrones para identificar y clasificar el aroma de las muestras medidas (Lozano, Arroyo, Santos, Cabellos, \& Horrillo, 2008; Quicazan, Díaz M., \& Zuluaga D., 2011).

Es necesario implementar técnicas de procesamiento una vez obtenidas las señales o patrones de los sensores de la nariz electrónica con el fin de interpretarlos y analizarlos de acuerdo con lo que se desea obtener y así construir modelos confiables de respuesta para predicción, identificación o clasificación, según sea el caso (Quicazan et al., 2011). Existen diferentes métodos analíticos con los cuales el investigador puede contar y su elección depende de los datos disponibles y del tipo de resultado que requiera. Entre los métodos más utilizados se encuentran los siguientes: el análisis gráfico (diagramas de barras y polares), análisis estadístico multivariado (análisis de componentes principales (PCA)), el análisis de función de discriminantes (DFA) y el análisis de clusters (CA), análisis de redes (redes neuronales artificiales (ANN)) y funciones de base radial (RBF)) (Ghasemi-Varnamkhasti et al., 2011; Rodríguez, Albarracín \& Delgado, 2011). 
La legislación alimentaria es cada vez más estricta en lo que respecta a la calidad y seguridad de los alimentos que se producen y comercializan, por lo que es necesario disponer de tecnologías adecuadas que permitan cumplir y verificar el cumplimiento de todos los requisitos legales en materia alimentaria. En este sentido, las características de los biosensores los convierten en excelentes instrumentos capaces de competir exitosamente en el mercado agroalimentario con otras tecnologías para contribuir al control de la calidad y seguridad alimentaria. Estos dispositivos pueden aplicarse para la trazabilidad alimentaria, el análisis de la composición de los alimentos, en la estimación de su vida útil y grado de frescura, e la detección de fraudes alimentarios (ej., sustitución de una especie animal por otra de menor valor económico, presencia de harinas cárnicas y de pescado en piensos, etc.) y la detección y cuantificación de compuestos xenobióticos (ej., aditivos, fármacos, plaguicidas, fertilizantes, dioxinas, PCBs, metales pesados, etc.), componentes de los alimentos (ej., antinutrientes, alérgenos, grasas, etc.), biotoxinas (ej., toxinas bacterianas, toxinas fúngicas 0 micotoxinas $y$ toxinas marinas) $y$ microorganismos alterantes y patógenos (bacterias, hongos, levaduras, virus $\mathrm{y}$ parásitos).
El análisis de componentes principales (PCA) es el método más común para visualizar y clasificar la estructura de datos multivariados. El PCA es una técnica quimiométrica bien conocida, que proyecta los datos en un hiperespacio reducido, definido por los componentes principales (Parellón, 2004). Este algoritmo cae dentro de las técnicas denominadas reconocimiento de patrones o técnicas de inteligencia artificial y permite reducir, representar y extraer información relevante al mismo tiempo y suele ser clasificado como un método no supervisado de reconocimiento de patrones, ya que su uso más extendido con las narices electrónicas se limita a representar bidimensionalmente un conjunto de medidas para ver si se pueden formar agrupaciones ("clusters") espontáneas entre las diferentes medidas realizadas. Este método construye una transformación lineal que escoge un nuevo sistema de coordenadas para el conjunto original de datos en el cual la varianza de mayor tamaño del conjunto de datos es capturada en el primer eje (llamado el Primer Componente Principal), la segunda varianza más grande es el segundo eje, y así sucesivamente. (Durán, 2005; Moreno, Caballero, Galán, Matía, \& Jiménez, 2009; Rojas, Comina, \& Solís, 2012; Zuluaga D, 2011). El uso de esta técnica permite la visualización de la estructura latente de datos mediante gráficas, cuyas interpretaciones permiten un entendimiento más profundo del que es 
posible obtener cuando se evalúan las variables en solitario, ya que permite analizar varias variables simultáneamente. EI PCA como análisis exploratorio es el punto de partida conveniente dentro de los métodos de datos multivariantes. (Esbensen \& Geladi, 2009). Otro método de análisis de datos multivariante muy utilizado es el de las Redes neuronales (ANN). Es un tipo de algoritmos inspirado en las redes neuronales biológicas, ajustan sus modelos de forma experimental, sin necesitar un conocimiento previo de las características de los datos que debe procesar ni de los mecanismos que lo producen. Este método ofrece una valiosa capacidad de mapeo no-lineal, muchos tipos de redes neuronales se han empleado hasta el momento para realizar análisis de clasificación con los datos de la nariz electrónica, incluidas las redes de Kohonen, el vector de aprendizaje (LVQ) y sus variaciones de backpropagation (BP) y el algoritmo ART. (Benito, Ortiz, Sánchez, Sarabia, \& Iniguez, 1999; Men, Liu, Pan, Wang, \& Zhang, 2011)

En la actualidad se han llevado a cabo múltiples investigaciones con la utilización de los sistemas multisensoriales como nariz y lengua electrónica con el fin de incursionar en el mejoramiento de las técnicas que permitan evaluar la calidad de los alimentos que se producen lo que ha ocasionado que en la última década se haya producido un rápido desarrollo comercial y tecnológico de los sentidos electrónicos. Las narices electrónicas son equipos desarrollados a los cuales se les atribuye múltiples aplicaciones en la industria agroalimentaria como son: valoración de la calidad en productos frescos (frutas, verduras, hortalizas, carne, leche y pollo crudo entre otros) y procesados (manufacturados), supervisión y control de operaciones básicas en la industria agroalimentaria (cocción, fermentación, mezclado, maduración, tostado entre otros), control de producto almacenado y estudio de su vida útil, evaluación del envejecimiento y maduración de vinos y otros productos de la industria alimentaria (quesos, productos cárnicos, frutas), monitorización de la interacción producto-empaque, la evaluación sensorial y de calidad de múltiples alimentos. (Correa, Barreiro, Ruiz-Altisent, \& Chamorro, 2005). De igual forma, los consumidores son cada vez más conscientes de los problemas de autenticación con ciertos grupos de alimentos y están dispuestos a pagar por productos más caros cuando el origen y los ingredientes están debidamente determinados. Debido a estos hechos, existe una creciente demanda entre las empresas y los institutos de dispositivos que permiten un análisis rápido de los productos alimenticios como son los sistemas multisensoriales que a pesar de las numerosas desventajas de los sensores electrónicos, como la calibración, 
la escasa selectividad y el complicado análisis de datos, estos dispositivos se utilizan cada vez más para el análisis de alimentos y en otras ramas de la vida cotidiana como en perfumería, en la industria farmacéutica, en operaciones forenses, ambientales y demás.

\section{CONCLUSIONES}

Las técnicas más utilizadas para la detección de pesticidas por su efectividad, confiabilidad, selectividad y sensibilidad son la cromatografía de Gases-Masas, HPLC, cromatografía de gases con detector de electrones entre otras múltiples técnicas. Independiente del tipo de analito y en especial del tipo de pesticida objeto de análisis en matrices alimenticias, el tratamiento de la muestra constituye la etapa más crítica para el análisis cuantitativo. Entre más etapas se involucren en el procedimiento, la probabilidad de perder los compuestos de interés aumenta. Sin embargo, una buena planeación de la metodología de extracción, concentración y cuantificación determina la fiabilidad de los datos obtenidos.

Aunque la cromatografía gaseosa cobra gran relevancia, sin duda alguna la cromatografía líquida de alta eficiencia, de resolución rápida constituye la técnica del futuro con la cual se podrá tener una aproximación muy grande a las concentraciones reales de un contaminante dentro de una matriz de interés. Pese a lo anterior, el desarrollo de un método cromatográfico constituye varias etapas que no solo consumen tiempo, sino, que demandan un amplio conocimiento químico y un trabajo riguroso y responsable. Poseer técnicas complementarias como los inmunoensayos y los biosensores contribuyen no solo a realizar monitoreos en tiempo real, sino, a tener indicadores previos antes de proceder con el desarrollo de una técnica de alta precisión y resolución.

Las industrias son cada vez más conscientes de los problemas que acarrea el que los alimentos que se comercializan, principalmente aquellos de origen perecedero como las frutas, no cumplan con los requisitos mínimos de calidad e inocuidad exigidos por la norma, por tal motivo, están dispuestos a invertir en métodos rápidos que permitan el análisis de componentes perjudiciales, como son los pesticidas. Al igual, los consumidores están dispuestos a pagar por productos más caros cuando son conscientes que su salud está en riesgo.

El origen y los avances en sistemas multisensoriales (narices y lenguajes electrónicos) para los ensayos de autenticidad alimentaria representan 
actualmente una alternativa para la detección de compuestos peligrosos en alimentos y debido a estos hechos, existe una creciente demanda entre empresas e institutos de dispositivos que permitan el análisis rápido de los productos alimenticios. Se utilizan varias técnicas y métodos para la evaluación de presencia o no de pesticidas en los alimentos. Uno de estos es por medio de las técnicas cromatográficas y los diferentes métodos de extracción que se utilizan para que sea confiable el análisis. La principal desventaja de este método es el hecho que requieren tiempos de análisis largos y representan alto costo de equipos y de mantenimiento, además del personal capacitado que se debe contratar para utilizarlos. En la industria, uno de los factores importantes que influyen en la elección del método analítico es el análisis. A pesar de las muchas desventajas de los sensores electrónicos, como calibración complicada, mala selectividad de los sensores y complicado análisis de datos, estos dispositivos se utilizan cada vez más para el análisis predictivo de alimentos y en otras ramas de la vida como la perfumería, la industria farmacéutica y las operaciones forenses. Además, existe una tendencia a combinar los datos de los sistemas multisensoriales (la nariz y la lengua electrónica) en evaluaciones de autenticidad que facilitan la evaluación del gusto y el olfato al mismo tiempo.

A medida que los alimentos van evolucionando y las posibles consecuencias que ello representa en la salud humana, irán apareciendo nuevas alternativas para la detección y análisis de los pesticidas en los alimentos y con el fin de mejorar las áreas de investigación, los fabricantes de instrumentos se enfocan en mejorar constantemente los materiales y el rendimiento de los sensores y del biosensor. El área de la robótica es la última tendencia para aplicar en la detección de compuestos extraños y perjudiciales en alimentos.

\section{REFERENCIAS BIBLIOGRÁFICAS}

Albert, L., \& Rendon-Von Osten, J. (1988). CONTAMINACIÓN POR COMPUESTOS ORGANOCLORADOS EN ALGUNOS. Rev. Saúde públ.,, 22:500-6.

Al-Saleh, I. (1994). Pesticides: a review article. J Environ Pathol Toxicol Oncol, 13, 151-161.
Ambrus , A., Füzesi, I., Susán, M., Dobi , D., Lantos, J., F. Korsós , I., et al. (2005). A cost-effective screening method for pesticide residue analysis in fruits, vegetables, and cereal grains. $J$. Environ. Sci. Heal. B., 40, 297-339.

Anastassiades , M., Lehotay, S., Stajnbaher, D., \& Schenck, F. (2003). 
Fast and easy multiresidue method employing acetonitrile extraction/partitioning and dispersive solid-phase extraction for the determination of pesticide residues in produce. Journal of AOAC International, 86(2), 412-431.

AOAC Official Method . (2007). esticide Residues in Foods by Acetonitrile Extraction and Partitioning with Magnesium Sulfate.

Araoud, M., Douki, W., Rhim, A., Najjar, M., \& Gazzah, N. (2007). Multiresidue analysis of pesticides in fruits and vegetables by gas chromatographymass spectrometry. Journal of

Environmental Science and Health Part B., 42(1), 179-187.

Araque Ordoñez, R. (2014). Evaluación de un Sistema de Enmascaramiento de Olor de Muestras de Ajo, mediante un sistema de Nariz Electrónica. Tesis de Master, Universidad Politécnica de Valencia, Escuela Técnica Superiorde Ingeniería Agronómica y del Medio Natural, Valencia.

Arienzo, M., Masuccio, A., \& Ferrara, L. (2013). Evaluation of sediment contamination by heavy metals, organochlorinated pesticides, and polyaromatic hydrocarbons in the Berre Coastal Lagoon (Southeast France). Arch. Environ. Contam. Toxicol, 65, 396406.
Arrieta A. y Quijano A., (2016). Identificación de hidrocarburos aromáticos policíclicos en muestras de ganado bovino de la vereda j 10 del municipio de Tibu. Revista @LIMENTECH CIENCIA Y TECNOLOGÍA ALIMENTARIA. ISSN 1692-7125. Volumen 14 No. 1, p. 84 - 93. Facultad de Ingenierías y Arquitectura. Universidad de Pamplona.

Banerjee, K., Utture , S., Dasgupta , S., Kandaswamy, C., Pradhan , S., Kulkarni, S., et al. (2012). Multiresidue determination of 375 organic contaminants including pesticides, polychlorinated biphenyls and polyaromatic hydrocarbons in fruits and vegetables by gas chromatographytriple quadrupole mass spectrometry. $J$. Chromatogr.A., 1270, 283-295.

Barbera, C. (1989). Pesticidas Agrícolas (4 ed.). Barcelona: Ediciones Omega.

Barbera, C. (1989). Pesticidas Agrícolas (cuarta edición). Omega.

Barron, M. G., Ashurova, Z. J., Kukaniev, M. A., Avloev, H. K., Jamshedov , J. N., Rahmatullova, O. S., et al. (2017, February 14). Residues of organochlorine pesticides in surface soil and raw foods from rural areas of the Republic of Tajikistan*. Environmental Pollution.

Bempah, C. K., Agyekum, A. A., Akuamoa , F., Frimpong, S., \& Buah-Kwofie, A. (2016). Dietary exposure to chlorinated pesticide residues in fruits and 
vegetables from Ghanaian markets. Journal of Food Composition and Analysis, 46, 103-113.

Bempah, C. K., Agyekum, A. A., Akuamoa, F., Frimpong, S., \& Buah-Kwofie, A. (2016). Dietary exposure to chlorinated pesticide residues in fruits and vegetables from Ghanaian markets. Journal of Food Composition and Analysis, 46, 103-113.

Benito, M. J., Ortiz, M. C., Sánchez, M. S., Sarabia, L. A., \& Iniguez, M. (1999). Typicfication of vinegars from Jerez and Rioja using classical chemometric techniques and neural network methods. Analyst, 124, 547-552.

Camino-Sánchez F. J., Zafra-Gómez A., Oliver-Rodríguez B., Ballesteros O., Navalon A., Crovetto G. y Vílchez J. L. (2011). UNE_EN ISO/IEC 17025:2005 accredited method for the determination of 121 pesticide residues in fruits and vegetables by gas chromatographytandem mass spectrometry. J. Food Compos. Anal. 24, 427-440.

Carabias-Martínez ， R., RodríguezGonzalo , E., Miranda-Cruz, E., Domínguez-Álvarez, J., \& HernándezMéndez, J. (2007). Sensitive determination of herbicides in food samples by nonaqueous CE using pressurized liquid extraction. Electrophoresis, 28, 3606-3616.

Carvajal Ferrer, A. A. (2013). Implementación de un método estocástico de busqueda acoplando técnicas de clasificación, Máquinas de Vectores de Soporte (SVM) para la selección de variables en sistemas Multisensoriales. Tesis Maestría, Universidad de Pamplona, Facultad de Ingenierías y Arquitectura, Pamplona.

Carvajal, L., Cucaita, G., Zuluaga, C., \& Quicazan, M. (2012). Uso de la Nariz Electrónica y panel sensorial para la evaluación de la calidad de la Mora. Vitae, 19(1), S60-S62.

Castro, R. (2005). Determinación de residuos de plaguicidas organofosforados en muestras de toma de de la ciudad de Bogota. Bogotá.

Cervera, M. I., Medina, C., Portolés, T., Pitarch, E., Beltrán, J., Serrahima, E., et al. (2010). Multi-residue determination of 130 multiclass pesticides in fruits and vegetables by gas chromatography coupled to triple quadrupole tandem mass spectrometry. Anal. Bioanal. Chem, 397, 2873-2891.

Chai, M. K., \& Tan, G. H. (2009). Validation of a headspace solid-phase microextraction procedure with gas chromatography-electron capture detection of pesticides residues in frits and vegetables. Food Chemistry, 117, 561-567.

Choi, M., Lee, I., \& Jung, R. (2016). Rapid determination of organochlorine pesticides in fish using selective pressurized liquid extraction and gas chromatography-mass spectrometry. Food Chem., 205, 1-8. 
Codex Alimentarius. (1996). Programa Conjunto FA/OMS sobre Normas Alimentarias Comisión del Codex Alimentarius. Roma.

Codex Alimentarius. (2008). www.fao.org. Retrieved Junio 25, 2016, from http://www.fao.org/fao-who-

codexalimentarius/standards/pestres $/ \mathrm{m}$ aterias-primas/es/

Columé, A., Cárdenas, S., Gallego , M., \& Valcárcel, M. (2001). "Semiautomatic multiresidue gas chromatographic method for the screening of vegetables for 25 organochlorine and pyrethrois pesticides. Analytica Chimica Acta., 153-162.

Cooper, J., \& Dobson, H. (2007). The benefits of pesticides to mankind and the environment. Crop Prot, 26, 1337-1348.

Correa, E. C., Barreiro, P., Ruiz-Altisent, M., \& Chamorro, C. (2005). Nariz electrónica ¿Herramienta para la calidad en la Industria Agroalimentaria? España.

Cortes, D., \& Hites, R. (2000). Detection of statistically significant trends in atmospheric concentrations of semivolatile compounds. Environ. Sci. Technol., 34, 2826-2829.

Cortes, J., Sánchez, R., Díaz-Plaza, E., Villen, J., \& Vásquez, A. (2006). Large volume GC Injection for the analysis of organophosphorus pesticides in vegetables using the through oven transfer adsorption desorption (TOTAD) Interface. J. Agric. Food Chem., 54.
Costa, L. G., Galli, C., \& Murphy, S. D. (1986). Toxicology of Pesticides: Experimental, clinical and regulatory perspectives. NATO ASI Series, 1-10.

Dimond, J. B., Getchell, A. S., \& Blease, J. A. (1971). Accumulation and Persistence of DDT in a Lotic Ecosystem. Journal of the Fisheries Research Board of Canada, 28(12), 1877-1882.

Dömötörová, M., \& Matisová, E. (2008). Fast gas chromatography for pesticide residue analysis. Journal of Chromatography A., 1207, 1-16.

Duodua, G. O., Goonetillekeb, A., \& Ayoko, G. A. (2016, April 1). Optimization of incell accelerated solvent extraction technique for the determination of organochlorine pesticides in river sediments. Talanta, 150, 278-285.

Duran, C. (2005). Diseño y optimización de los subsistemas de un sistema de olfato electrónico para aplicaciones agroalimentarias e industriales. Tarragona.

Duran-Acevedo, C. M., Gualdron-Guerrero, O. E., \& Hernandez-Ordoñez, M. (2014). Nariz electrónica para determinar el índice de madurez del tomate de árbol (Cyphomandra Betacea Sendt). Ingeniería Investigación y Tecnología, 15(3), 351-362.

Esbensen, K. H., \& Geladi, P. (2009). Principal Component Analysis: Concept, Geometrical Interpretation, 
Mathematical Background, Algorithms, History, Practice. Elsevier.

Escalona, A., Galarraga, F., Fernández, R., Centeno, A., Velásquez, J., \& Pérez, G. (2012). Desarrollo de métodos no convencionales para la extracción de fenantreno y pireno en partículas sedimentadas. Rev. Fac. Ing. UCV, 27(1), 41-48.

Escuderos M., M., Santos D., M., Rubio B., S., \& Polo D., L. M. (2003). Direct determination of monolinuron, linuron and chlorbromuron residues in potato samples by gas chromatography with nitrogen-phosphorus detection. $J$. Chromatogr. A., 1011, 143-153.

Farajzadeh, M. A., Asghari, A., \& Feriduni, B. (2016, May). An efficient, rapid and microwave-accelerated dispersive liquid-liquid microextraction method for extraction and pre-concentration of some organophosphorus pesticide residues from aqueous samples. Journal of Food Composition and Analysis, 48, 73-80.

Farajzadeh, M. A., Yadeghari, A., \& Khoshmaram, L. (2017, March). Combination of dispersive solid phase extraction and dispersive liquid-liquid microextraction for extraction of some aryloxy pesticides prior to their determination by gas chromatography. Microchemical Journal, 131, 182-191.

Farina, Y., Abdullah, M. P., Bibi, N., \& Khalik, W. M. (2017). Determination of Pesticide Residues in Leafy Vegetables at Parts per Billion Levels by a Chemometric Study Using GC-ECD in Cameron Highlands, Malasya. Food Chemistry.

Fenik, J., ankiewicz, M., \& Biziuk, M. (2011). Properties and determination of pesticides in fruits and vegetables. Trends in Analytical Chemistry, 30(6).

Ferrer I., T. E., \& Zweigmenbaum, J. (2011). LC/TOF-MS analysis of pesticides of fruits and vegetables the emerging of accurate mass in inambiguous indetification of pesticides in food. Mass Spectrometry in Food Safety. Series Methods in Molecular Biology, 747, 193-218.

Ferrer, A. (2003). Intoxicación por plaguicidas. Anales del Sistema Sanitario de Navarra, 26(1), 155-171.

Figaro. (2016). Figaro USA,INC. Retrieved Julio $\quad 15, \quad 2016, \quad$ from http://www.figarosensor.com/

Food Agriculture Organization United Nations (FAO). (2008). International code of conduct on the distribution and use of pesticides. Roma, p.28.

Frank, M. (1999). Solid State Chemical Sensors . Sensors for Food Applications.

Fuentes E., B. M. (2009). Microwaveassisted extraction at atmospheric pressure coupled to different clean-up methods for the determination of organophosphorus pesticides in olive and avocado oil. $J$. Chromatogr. A., 1216, 8859- 8866. 
Gancarz, M., Wawrzyniak, J., GawrysiakWitulska, M., Wiącek, D., Nawrocka, A., Tadla, M., et al. (2017). Application of electronic nose with MOS sensors to prediction of rapeseed quality. Measurement.

García Cambero , J. P. (2002). Estado actual de la contaminación por metales pesados y pesticidas organoclorados en el parque natural de Monfragûe. Tesis Doctoral, Universidad de Extremadura, España.

Garrido, L. R. (1988). Toxicología de Pesticidas. méxico: Garrido-Lestache.

Gualdron, O. (2006). Desarrollo de diferentes métodos de selección de variables para sistemas multisensoriales. Tesis de Doctorado, Universitat Rovira I Virgili, Departament D`Enginyeria Electrònica Elèctrica I Automàtica, Tarragona.

Gualdron, O., Duran, C., Ortiz, J., \& Araque, J. (2014). Implementation of a neural model in a hardware device(FPGA) for the classification of chemical compounds in a multisensory (E-nose). Rev. Colomb. Tecnol. Av, 2(24), 127-133.

Guerrero, J. A. (2003). Estudio de residuos de plaguicidas en frutas y hortalizas en áreas específicas de Colombia. Agronomía Colombiana, 21(3), 198-209.

Guerrero, M., \& Herrera, J. (2016). Desarrollo, validación y estimación de incertidumbre de un método cromatográfico para determinar residuos de plaguicidas organofosforados y cipermetrina en tomate. Revista Agua y Conocimiento CIRA -UNAN, 2(1), 19-33.

Hirahara , Y., Kimura, M., Inoue , T., Uchikawa, S., Otani, S., Haganuma , A., et al. (2005). Validation of multiresidue screening methods for the determination of 186 pesticides in 11 agricultural products using gas chromatography (GC). J. Health Sci., 51, 617-627.

Hong, X., \& Wang, J. (2014). Detection of adulteration in cherry tomato juices based on electronic nose and tongue: comparison of different data fusion approaches. Journal of Food Engineering, 126, 89.97.

Kafilzadeh, F. (2015). Assessment of Organochlorine Pesticide Residues in Water, Sediments and Fish from Lake Tashk, Iran. Achievements in the Life Sciences, 9(2), 107-11.

Leadprathom, N., Parkpian, P., Satayavivad, J., Delaune, R., \& Jugsujinda, A. (2009). Transport and deposition of organochlorine pesticides from farmland to estuary under tropical regime and their potential risk to aquatic biota. J. Environ. Sci. Health Part B, 44, 249-261.

Lehotay, S. J. (2005). Validation of a fast and easy method for the determination of residues from 229 pesticides in fruits and vegetables using gas and liquid chroma- tography and mass 
spectrometric detection. J. AOAC Int., 88, 595-614.

Li, J.-W., Wang, Y.-L., Yan, S., Li, X.-J., \& Pan, S.-Y. (2016, February 1). Molecularly imprinted calixarene fiber for solid-phase microextraction of four organophosphorous pesticides in fruits. Food Chemistry, 192, 260-267.

Liu, H., Kong, W., Gong, B., Miao, Q., Qi, Y., \& Yang, M. (2015, January). Rapid analysis of multi-pesticides in Morinda officinalis by GC-ECD with accelerated solvent extraction assisted matrix solid phase dispersion and positive confirmation by GC-MS. Journal of Chromatography $B, 974,65-74$.

Lozano, J., Arroyo, T., Santos, J., Cabellos, J., \& Horrillo, M. (2008, Jul 28). Electronic Nose for Wine Ageing Detection. Sensors Actuators B-Chem, 133(1), 180-186.

Ma, J., Pan, L.-b., Yang, X.-y., Liu, X.-I., Tao, S.-y., Zhao, L., et al. (2016, august 25). DDT, DDD, and DDE in soil of Xiangfen County, China: Residues, sources, spatial distribution, and health risks. (H. E. Boer, Ed.) Chemosphere, 163, 578-583.

Mannino, S., Benedetti, S., Buratti, S., \& Cosio, M. S. (2005). Gas and liquid sensors for quality control in food processing. Food Chemistry.

Manviri , R., Uma , S., \& Vidhisha , J. (2017). Recent strategies for removal and degradation of persistent \& toxic organochlorine pesticides using nanoparticles: A review. Journal of Environmental Management, 208-222.

Marrs, T., \& Ballantyne, B. (2004).

Pesticide Toxicology and International Regulation. John Wiley \& Sons.

Matsumura, F. (1985). Toxicology of Pesticides. New York: Segunda Edición,Plenum Press.

Men, H., Liu, H., Pan, Y., Wang, L., \& Zhang, H. (2011). Electronic Nose Based on an Optimized Competition Neural Network. Sensors, 11, 50055019.

Mendoza Patiño, N., \& León Rodríguez, J. A. (2005). Prohibición del uso del lindano. Rev Fac Med UNAM, 48(3), 113-114.

Mijangros R., O. F. (2010). Optimización de métodos de extracción acelerados mediante energías auxiliares $y$ caracterización de la fracción fenólica de Prosthechea karwinskii y Prosthechea varicosa. Instituto Politécnico Nacional, Oxaca.

Mohamed, H., EL-Saeid, Saleh , A., \& ALDosari. (2010, July). Monitoring of pesticide residues in Riyadh dates by SFE, MSE, SFC, and GC techniques. Arabian Journal of Chemistry, 3(3), 179_ 186.

Montaño Garcés, M., \& Guerrero Dallos, J. A. (2001). Validación de una Metodología Multiresiduo para la determinación de residuos plaguicidas en fresa (fragaria spp) por 
Cromatografía de Gases. Revista Coombiana de Química, 30(1).

Moon, H.-B., Kim, H.-S., Minkyu, C., Jun, Y., \& Hee-Gu, C. (2009). Human health risk of poly chlorinated biphenyls and organochlorine pesticides resulting from sea food consumption in South Korea, 2005-2007. Food Chem. Toxicol, 18191825.

Moreno, I., Cballero, R., Galan, R., Matía, F., \& Jiménez, A. (2009). La Nariz Electrónica: Estado del Arte. Revista Iberoamericana de Automática e Informática Industrial, 6(3), 76-91.

Moreno, M. (2001). Evaluación de la residualidad de plaguicidas en el repollo (Brassica Oleracea var'capitata) en el departamento de Cundinamarca. Trabajo de Grado, Universidad Nacional de Colombia, Cundinamarca, Bogotá.

Moses , M. (1993). Pesticides. Williams \& Wilkin.

Muñoz, V. (1992). Situación y perspectivas de la horticultura en Colombia. Primer curso de horticultura de clima frio. Conferencia. ICA . Tibaitatá. Mosquera. Murcia, A. M., \& Stashenko, E. (2008). Determinación de Plaguicidas Organofosforados en Vegetales producidos en Colombia. Agro Sur, 36, 71-81.

Musaiger , A. O., Al-Jedah , J. H., \& D' Zousa, R. (2008). Ocurrence of contaminants in foods commonly consumed in Bahrain. Food Control, 19, 854-861.
OMS, OPS, División Salud y Ambiente. (1993). Plaguicidas y salud en las Américas. Washington.

Ongo, E., Falasconi, M., Sberveglieri, G., Antonelli, A., Montevecchi, G., Sberveglieri, V., et al. (2012). Chemometric Discrimination of Philippine Civet Coffee Using Electronic Nose and Gas Chromatography Mass Spectrometry. Procedia Engineering, 47, 977-980.

Organización Mundial de la Salud (OMS), Organización Panamericana de la Salud (OPS), Centro Panamericano de Ecología Humana y Salud. (1990). Plaguicidas Organoclorados. México.

Ortiz S., J. E. (2016). Detección de Plaguicidas (organoclorados) en alimentos frescos (Frutas) usando una nariz electrónica. Tesis de Maestría, Universidad de Pamplona, Norte de Santander, Pamplona.

Ortiz, J., Gualdron, O., \& Duran, C. (2106, June). Detection of pesticide in fruits using an electronic nose. Australian Journal of Basic and Applied Sciences, 10(10), 107-113.

Pabon Mora, C. (2016). Evaluación del grado de adulteración del café (coffea arabica) tostado y molido con habas (Vicia faba). Trabajo de investigación de Maestría, Universidad de Pamplona, Facultad de Ingenierías y Arquitectura, Maestria en Ciencia y Tecnología de Alimentos. Pamplona, Colombia. 
Pabuena, D. E., Ortiz, I. C., Lopez, J., Orozco, L. J., Quijano, A., Pardo, E., et al. (2015, Septiempbre). Actividad genotóxica por esxtracto de fresa fumigada con pesticidas en Pamplona, Norte de Santander, Colombia. Universidad, Ciencia y Tecnología, 19(76), 111-117.

Páez, M. I., \& Martínez, J. M. (2015). Implementación de la metodología QuEChERS en el análisis de residuos de plaguicidas en maíz blanco (Zea mays). Temas Agrarios, 20(2), 30-42.

Parellón, O. B. (2004). Diseño y realizción de una nariz electrónica para maduración de quesos. España.

Pérez, M. A., Navarro, H., \& Miranda, E. (2013). Residuos de Plaguicidas en Hortalizas: problemática y riesgo en México. Rev. Int.Contam. Ambie(29), 45-64.

Pérez, R., Salas, J., \& Amaro, R. (2015). Comparación de dos métodos de acción para la determinación de pesticidas anoclorados y organofosforados en fresas. Scientifc Journal from the Experimental Faculty of Sciences, 23(3), 158-167.

Persaud, K., \& Dodd, G. (1982). Analysis of discrimination mechanisms of the mammalian olfactory system a model nose. Nature, 299, 352-355.

Poustka , J., Holadová , K., \& Hajšová , J. (2003). Application of supercritical fluid extraction in multi-residue pesticide analysis of plant matrices. Eur. Food. Res. Technol., 216, 68-74.

Quu, S., Wang, J., \& Gao, L. (2014). Discrimination and Characterization of Strawberry Juice Based on Electronic Nose and Tongue: Comparison of Different Juice Processing Approaches by LDA, PLSR, RF, and SVM. Journal of Agricultural and Food Chemistry, 62, 6426-6434.

Quicazan S, M. C., Diaz M, A. C., \& Zuluaga D, C. M. (2011). La Nariz Electrónica, una novedosa herramietna para el control de procesos y calidad en la Industria Agroalimentaria. Rev. Vitae, 18(2), 209-217.

Quicazan, M. C., Díaz M., A. C., \& Zuluaga D., C. M. (2011). La Nariz electrónica, una novedosa herramienta para el control de procesos y calidad en la industria agroalimentaria. Vitae, Rev.de la Fac. de Química Farmacéutica, 18(2), 209-217.

Raldúa Pérez, D. (1995). Estudio de la Contaminación por plaguicidas organclorados y mercurio en la ictiofauna de los ríos altoaragoneses Gállego y Cinca". Tesis Doctoral.

Ramirez, J. A., \& Lacasaña, M. (2001). Plaguicidas: clasificación, uso, toxicología y medición de la exposición. Arch Prev Riesgos Labor, 4(2), 67-75.

Ramírez, J., \& Lacasaña, M. (2001). Plaguicidas: clasificación, uso, toxicología y medición de la exposición. Arch Prev Riesgos Labor, 4(2), 67-75. 
Rejczak , T., \& Tuzimski, T. (2017, February).

QuEChERS-based

extraction with dispersive solid phase extraction clean-up using PSA and ZrO2-based sorbents for determination of pesticides in bovine milk samples by HPLC-DAD. Food Chemistry, 217, 225233.

Revista Dinero. (n.d.). www.dinero.com. Retrieved 10 11, 2016, from http://www.dinero.com/pais/articulo/exp ortaciones-colombianas-fruta/209741

Rissato, S. R., Galhiani, M. S., \& Gerenutti, M. (2005). Multiresidue Method for Determination of Pesticides in Fruits using superficial Fluid Extraction. Información Tecnológica, 16(6), 13-19.

Rodríguez-Gonzalo , E., CarabiasMartínez, R., Miranda-Cruz, E., Domínguez-Alvarez, J., \& HernándezMéndez, J. (2009). Ultrasonic solvent extraction and nonaqueous CE for the determination of herbicide residues in potatoes. J. Sep. Sci., 32, 575-584.

Rodríguez-Méndez, M. L. (2001). Análisis sensorial de alimentos. Nariz Electrónica. Investigación química.

Roeck, F., Barsan, N., \& Weimar, U. (2008). Electronic nose: Current status and future trend. Chemical Reviews, 108, 705-725.

Rojas, M., Comina, G., \& Solìs, J. (2012). Prototipo de una nariz electrónica. 75 80.

Romanic, S. H., Klincic, D., KljakovicGašpic, Z., Kusak, J., Reljic, S., \&
Huber, Đ. (2015). Organochlorine pesticides and polychlorinated biphenyl congeners in wild terrestrial mammals from Croatia: Interspecies comparison o residue levels and compositions. Chemosphere, 137, 52-58.

Sánchez, R. (2002). Plaguicidas y fitosanitarios. Memorias. XIII Congreso Nacional Farmacéutico. Granada, España.

Sapahin, H. A., Makahleh , A., \& Saad, B. (2015). Determination of organophosphorus pesticide residues in vegetables using solid phase microextraction coupled with gas chromatography-flame photometric detector. Arabian Journal of Chemistry. Sliwinska, M., Wisniewska, P., Dymerski, T., Wardencki, W., \& Namiesnik, J. (2016). Advances in Electronic Noses and Tongues for Food Authenticity Testing. Poland.

Socas-Rodríguez, B., González-Sálamo, J., Herrera-Herrera, A. V., HernándezBorges, J., \& Rodríguez-Delgado, M. A. (2017, February 20). Recent Advances and Developments in the QuEChERS Method. Comprehensive Analytical Chemistry.

Thomas, A., Leontjew Toms, L. M., Harden, F. A., Hobson, P., White, N. M., Mengersen, K. L., et al. (2017). Concentrations of organochlorine pesticides in pooled human serum by age and gender. Environmental Research, 154, 10-18. 
Thomas, A., Tomsb, L.-M. L., Hardenc, F. A., Hobson, P., White, N. M., Mengersen, K. L., et al. (2017). Concentrations of organochlorine pesticides in pooled human serum by age and gender. Environmental Research, 154, 10-18.

Trtic-Petrovic, T., Dordevic, J., Dujakovic, N., Kumric, K., Kavasiljevic, T., \& Lausevic, M. (2010, Jul). Determination of selected pesticides in environmental water by employing liquid-phase microextraction and liquid chromatography-tandem mass spectrometry. Anal Bioanal Chem., 397(6), 2233-43.

Van Pinxteren , M., Bauer, C., \& Popp , P. (2009). High performance liquid chromatography-tandem mass spectrometry for the analysis of 10 pesticides in water: A comparison between membrane-assisted solvent extraction and solid phase extraction. J. Chromatogr. A., 1216, 5800-5806.

Velasco, R. J., Villada, H. S., \& Carrera, J. E. (2007). Aplicaciones de los Fluidos Supercríticos en la Agroindustria. Información Tecnológica, 18(1), 53-65.

Wang, H., Ding, J., \& Ren, N. (2016, January). Recent advances in microwave-assisted extraction of trace organic pollutants from food and environmental samples. TrAC Trends in Analytical Chemistry, 75, 197-208.

Wang, Y. Q., Wang , Y., Huo , X. X., \& Zhu, Y. C. (2015). Why some restricted pesticides are still chosen by some farmers in China? empirical evidence from a survey of vegetable and apple growers. Food Control, 51, 417-424.

Wanwimolruk, S., Kanchanamayoon, O., Phopin, K., \& Prachayasittikul, V. (2015). Food safety in Thailand 2 : pesticide residues found in Chinese kale (Brassica oleracea), a commonly consumed vegetable in Asian countries. Science Total Enviroment, 532, 447455.

Winter, C. K., \& Jara, E. A. (2015). Pesticide food safety standards as companions to tolerances and maximum residue limits. Journal of Integrative Agriculture, 14(11), 2358-2364.

Winter, C. K., \& Jara, E. A. (2015, November). Pesticide food safety standards as companions to tolerances and maximum residue limits. Journal of Integrative Agriculture, 14(11), 23582364.

X. Yang, H. Zhang, Y. Liu, J. Wang, Y.C. Zhang, A.J. Dong, et al. (2011, July 15). Multiresidue method for determination of 88 pesticides in berry fruits using solidphase extraction and gas chromatography-mass spectrometry: Determination of 88 pesticides in berries using SPE and GC-MS. Food Chemistry, 27(2), 855-865.

Yohannes, Y. B., Ikenaka, Y., Nakayama , S. M., Mizukawa, H., \& Ishizuka, M. (2016). DDTs and other organochlorine pesticides in tissues of four bird species 
from the Rift Valley region, Ethiopia.

Science of the Total Environment.

Zhang, W. J. (2008). A forecast analysis on world population and urbanization process. Environ. Dev. Sustain, 10, 717730.

Zhao, X., Zhou, Y., Kong, W., Gong, B., Chen, D., Wei, J., et al. (2016, Apri 1 ). Multi-residue analysis of 26 organochlorine pesticides in Alpinia oxyphylla by GC-ECD after solid phase extraction and acid cleanup. Journal of Chromatography B, 1017-1018, 211220.

Zuluaga D, C. M. (2011). Análisis Estadístico Multivariado: una Herramienta Estratégica para el Control de Procesos y Calidad en la Industria Agroalimetnaria. Publicaciones e Investigacion, 5. 